\title{
Imatinib mesylate alters the expression of genes related to disease progression in an animal model of uveal melanoma
}

\author{
Bruno F. Fernandes ${ }^{\mathrm{a}, *}$, Sebastian Di Cesare ${ }^{\mathrm{a}}$, Rubens Neto Belfort ${ }^{\mathrm{a}, \mathrm{b}}$, Shawn Maloney ${ }^{\mathrm{a}}$, \\ Claudia Martins $^{\mathrm{a}}$, Enzo Castiglione ${ }^{\mathrm{a}}$, Jordan Isenberg ${ }^{\mathrm{a}}$, Daniel Abourbih ${ }^{\mathrm{a}}$ and Emilia Antecka ${ }^{\mathrm{a}}$ \\ and Miguel N. Burnier Jr. ${ }^{\mathrm{a}, \mathrm{b}}$ \\ ${ }^{a}$ Department of Ophthalmology and Pathology, The McGill University Health Center \& Henry C. Witelson \\ Ocular Pathology Laboratory, Montreal, Canada \\ ${ }^{\mathrm{b}}$ Department of Ophthalmology, Federal University of Sao Paulo - UNIFESP/EPM, São Paulo, Brazil
}

\begin{abstract}
Imatinib mesylate (IM) is a compound that inhibits both BCR-ABL tyrosine kinase and c-kit receptors. Tyrosine kinases are important in cellular signaling and mediate major cellular processes such as proliferation, differentiation, apoptosis, attachment, and migration. Twenty-six albino rabbits were injected with $1 \times 10^{6}$ human uveal melanoma (UM) cells (92.1) into the suprachoroidal space. Animals were immunosuppressed (cyclosporin A) over the course of the 12-week experiment and divided into two groups $(n=13)$. The experimental group received IM once daily by gavage while the control group received a placebo. One animal per group was sacrificed every week after the 2 nd week. Upon necropsy, organs were harvested for histopathological examination. Cells from the primary tumors were recultured and tested in proliferation and invasion assays. A PCR array was used to investigate the differences in expression of 84 genes related to tumor metastasis. In the treated group, 4 rabbits developed intraocular tumors, with an average largest tumor dimension (LTD) of $2.5 \mathrm{~mm}$ and 5 animals reported metastatic disease. Whereas 6 rabbits in the control group developed intraocular tumors, with an average LTD of $5.8 \mathrm{~mm}$ and 6 animals reported metastatic disease. The recultured cells from the treated group demonstrated lower proliferation rates and were less invasive $(p<0.001)$. The PCR array showed differences in expression of genes related to metastasis. Notably, there was 290-fold increase in SERPINB5, a tumor suppressor gene, and a 10-fold higher expression of KISS1, a metastasis suppressor gene, in the treated group. Proangiogenic genes such as VEGFA, PDGFA and PDGFB were downregulated in the treated group. To the best of our knowledge, this is the first report detailing the altered expression of specific genes in UM cells after treatment with IM.
\end{abstract}

\section{Introduction}

Metastatic uveal melanoma (UM) still presents a challenge to ophthalmologists and oncologists.

\footnotetext{
${ }^{1}$ Part of this work was presented as a poster at the 2009 ARVO meeting.

*Corresponding author: Bruno F. Fernandes, 3775 University Street, Room 216, Montreal, Quebec H3A-2B4, Canada. Tel.: +1 5143987192 ext. 00384; Fax: +1 514398 5728; E-mail: bruno. mtl@gmail.com.
}

Despite successful control of the primary tumor, survival rates have remained markedly unchanged. Once metastases are detected, prognosis is poor and almost every patient dies within a year [1]. Several centers have tried different compounds, but the need for a suitable and effective drug that would actually improve the survival of metastatic melanoma patients is desirable.

Imatinib mesylate (IM) is a compound that inhibits various tyrosine kinases includings c-kit [2] and BCRABL [3]. Tyrosine kinases are important in cellular signaling and mediate major cellular processes such 
as proliferation, differentiation, apoptosis, attachment, and migration [4]. Previous work from our laboratory has demonstrated that $78 \%$ of UM express c-kit. Moreover, treatment of UM cell lines with IM inhibits proliferation and their invasive abilities [5]. In this study, we therefore aimed to assess the in vivo effects of IM in an animal model of UM.

\section{Methods}

The animal model was carried out in compliance with the Association of Research in Vision and Ophthalmology Statement for the Use of Animals in Ophthalmic and Vision Research. The approval of both the Animal Care Committee and the Ethics Subcommittee at McGill University was obtained prior to all experiments.

\subsection{Animals}

Twenty-six male New Zealand albino rabbits (Charles River Canada, St-Constant, Québec, Canada) were randomly divided into two groups, control and experimental, with mean initial weights of $2.73 \pm$ $0.11 \mathrm{~kg}$ and $2.79 \pm 0.13 \mathrm{~kg}$ respectively. The animals were immunosuppressed using daily intramuscular injections of cyclosporin A (CsA; Sandimmune $50 \mathrm{mg} / \mathrm{ml}$, Novartis Pharmaceuticals Canada Inc., Dorval, Québec, Canada) in order to avoid rejection of the human cells. CsA administration was maintained throughout the 12-week experiment to prevent tumor regression. The dosage schedule recommended in previous studies [6-8] was employed: $15 \mathrm{mg} / \mathrm{kg} / \mathrm{day}, 3$ days before cell inoculation and during 4 weeks thereafter, followed by $10 \mathrm{mg} / \mathrm{kg} /$ day during the last 8 weeks of the experiment [9]. CsA doses were adjusted weekly according to the animal weight to compensate for decreased animal weight and avoid possible toxicity.

\subsection{Cell lines}

The 92.1 primary human uveal melanoma cell line [10], kindly provided by Dr. Antonia Saornil from the Instituto Universitario de Oftalmobiología Aplicada (IOBA), University of Valladolid, was used. One million cells (cellular viability greater than $98 \%$, determined by the trypan blue exclusion test) were suspended in $0.1 \mathrm{ml}$ of RPMI-1640 media (Invitro- gen, Burlington, Ontario, Canada) and injected into the suprachoroidal space of the right eye of each rabbit according to a previously described technique [6-9].

\subsection{Drug administration}

Dose calculation for rabbits was done using the body surface area (BSA) normalization method. Based on that, we administered a dose of $260 \mathrm{mg} / \mathrm{m}^{2}$ of IM (Novartis Pharma AG, Basel, Switzerland) which was usually $18-20 \mathrm{mg} / \mathrm{kg}$ depending on the animal's weight. Doses were adjusted accordingly as the animals lost weight over the course of the experiment to avoid toxicity. The dose used is the dose recommend for treatment in children and equivalent to $476 \mathrm{mg}$ in a $70 \mathrm{~kg}$ adult.

\subsection{Histopathological studies}

One animal per group was euthanized per week starting on the second week after the inoculation of cells. The selection criterion was based on the appearance of the animal, signs of CsA toxicity and veterinary recommendations. The remaining rabbits of each group $(N=3)$ were sacrificed at the end of the experiment. The method of euthanasia was exsanguination following anesthesia using intramuscular ketamine-xylazine ( $35 \mathrm{mg} / \mathrm{kg}-5 \mathrm{mg} / \mathrm{kg}$ ). An autopsy was performed on every animal that was sacrificed. The enucleated eyes and other organs with possible metastatic disease such as lungs, livers and kidneys were collected, macroscopically examined and preserved in $10 \%$ phosphate buffered formalin. Formalin-fixed, paraffin-embedded sections of the collected specimens were stained with Hematoxylin and Eosin (H\&E) for histopathological assessment.

\subsection{In vitro assays}

The eye of each rabbit was processed prior to formalin fixation in order to acquire fresh tumor samples. Cell harvesting and reculturing was successful in 3 animals from each group. Cells were cultured in a 6-well plate in 5\% fetal bovine serum (FBS) supplemented RPMI and grown to confluence before seeding for proliferation and invasion assay experiments. The 
Sulforhodamine-B based assay kit (TOX-6, SigmaAldrich, St. Louis, Missouri, USA) was performed according to the National Cancer Institute protocol [11]. Recultured cells obtained from primary tumors were seeded in a 96-well plate at a concentration of $2.5 \times 10^{3}$ cells per well, with six wells per cell line. Cells were allowed to adhere overnight and incubate for 48 or 72 hours. Following both the 48 and 72 hour incubation periods, cells were fixed to the bottom of the wells using a solution of $50 \%$ Trichloroacetic acid (TCA) (Fisher Scientific Company, Ottawa, Ontario, Canada) for 1 hour at $4^{\circ} \mathrm{C}$. Plates were then rinsed with distilled water to remove the TCA and excess media and were air-dried. The Sulforhodamine-B dye solution was then added to each well and allowed to stain for 30 minutes. The Sulforhodamine-B solution was subsequently removed by washing with a $1 \%$ acetic acid solution and once more allowed to air dry. The dye that had become incorporated into the fixed cells at the bottom of the wells was solubilized in a $10 \mathrm{mM}$ solution of Tris base solution. The absorbance of the solute was measured using a microplate reader at a wavelength of $565 \mathrm{~nm}$.

For the invasion assay, a modified Boyden chamber consisting of polyethylene terephthalate membrane with $8 \mu \mathrm{m}$ diameter pores precoated with Matrigel, an artificial basement membrane (BD, Mississauga, Ontario, Canada), was used [12]. A polyethylene terephthalate membrane without Matrigel was used as a control. Briefly, $1.25 \times 10^{5}$ cells from both groups were added to the upper chamber in RPMI 1640 with $0.1 \%$ FBS. RPMI 1640 with $10 \%$ fetal bovine serum was added to the lower chamber as a chemoattractant. The chambers were then incubated at $37^{\circ} \mathrm{C}$ in $5 \% \mathrm{CO}_{2}$-enriched atmosphere for $48 \mathrm{~h}$ to allow for cellular invasion through the Matrigel. Non-invading cells were removed from the upper chamber by gently wiping the surface of the membrane with a moist cotton swab. Membranes were removed and then stained using a Diff-Quick staining set. Stained cells were counted microscopically in 20 high-powered $(400 \times)$ fields. Only cells whose nuclei had completely invaded through the membrane were counted. Each experimental condition was done in triplicate and the average number of invading cells was then calculated. Percentage invasion was determined using the following formula: $\%$ invasion = (mean number of cells invading through the Matrigel/mean number of cells migrating through control polyethylene terephthalate membrane) multiplied by one hundred.

\subsection{Statistical analysis}

The differences in proliferation and invasion rates for each uveal melanoma cell line from each rabbit were determined using the ANOVA test. A $p$-value of less than 0.05 was considered statistically significant. Calculations were computer-based using SPSS version 11.5 (SPSS Inc., Chicago, Illinois, USA).

\subsection{PCR array}

The Human Tumor Metastasis RT $^{2}$ Profiler PCR Array (Qiagen, Mississauga, Ontario, Canada) was employed in this study. In order to conform to the Bio-Rad Opticon Chromo4 thermocycler.Plate (MJ Research, Waltham, Massachusetts, USA) array format $\mathrm{D}$ was used. The array includes 84 genes known to contribute to or prevent metastatic disease. In addition, five housekeeping genes, a genomic DNA control, and three positive controls are included to ensure high quality data normalization across samples. Two primary tumors, one from each group, were chosen for this experiment, (R6, R27). These two specific rabbits were chosen because they were the ones that had the longest clinical endpoint throughout the entire 12week experiment. Total RNA was extracted from these samples using the RNeasy Mini Kit (Qiagen) following the protocol for isolation from animal cells. The $\mathrm{RT}^{2}$ First Strand Kit (Qiagen) was used in order to produce a cDNA library of the total RNA extracted. The cDNA was later processed to perform the real-time PCR array mentioned above.

\section{Results}

\subsection{Tumor development}

In the treated group, 4 rabbits developed intraocular tumors, with an average largest tumor dimension (LTD) of $2.5 \mathrm{~mm}$ and 5 animals reported metastatic disease. Whereas 6 rabbits in the control group developed intraocular tumors, with an average LTD of $5.8 \mathrm{~mm}$ and 6 animals reported metastatic disease. Metastases were seen only in the lungs (Fig. 1). Csa toxicity was generally low and not significant enough to bias the study. Because of the deterioration of the animal's condition were primarily due to tumor burden, the sequential sac- 

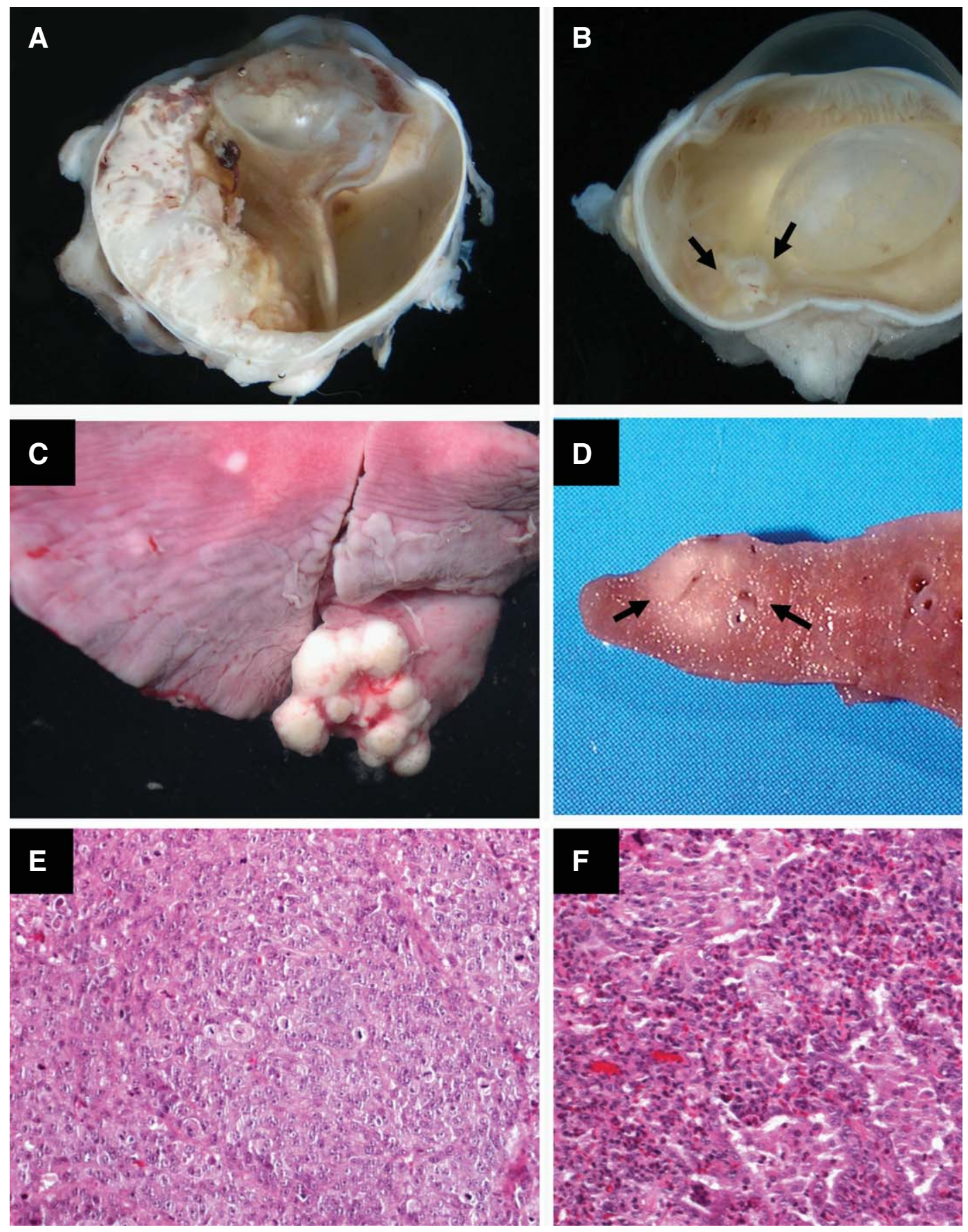

Fig. 1. Gross examination of post-mortem specimens: A) Enucleated eye from an animal of the control group harboring a large whitish intraocular tumor occupying almost half of the vitreal cavity. B) An eye from the group treated with Imatinib mesylate showing a much smaller tumor (arrows). C) White nodules representing metastatic dissemination to the lung. D) A cross-section of the lung showing a metastatic nodule (arrows). Microscopic images: E) Intraocular tumor, epithelioid cell-type with several mitotic figures $(\mathrm{H} \& \mathrm{E}, \times 200)$. F) Metastatic nodule revealing the malignant cells interspersed with an inflammatory infiltrate $(\mathrm{H} \& \mathrm{E}, \times 200)$. 
rifice of animals roughly reproduced the natural history of this particular animal model.

\subsection{In vitro assays}

The recultured cells from the treated group showed lower proliferation rates after 24 and 48 hours of incubation $(p<0.001)$. Cells from treated animals were markedly less invasive $(p<0.001)$ (Fig. 2).

\section{3. qPCR array}

All 84 genes present on the Human Tumor Metastasis RT ${ }^{2}$ Profiler PCR Array panel were successfully amplified in the primary tumor from both treated and untreated rabbits (R6, R27). When both samples were analyzed in comparison, with the SA Biosciences software provided, a list of genes were significantly different between the treated and control rabbits $( \pm 2$ fold relative change) (Table 1).

\section{Discussion}

The average life expectancy of UM patients that develop metastasis is as short as 3.6 months [13]. When patients are screened regularly and metastasis is discovered before the onset of symptoms, survival is longer but most likely due to lead-time bias [14-16]. Various studies have supported this theory by show-
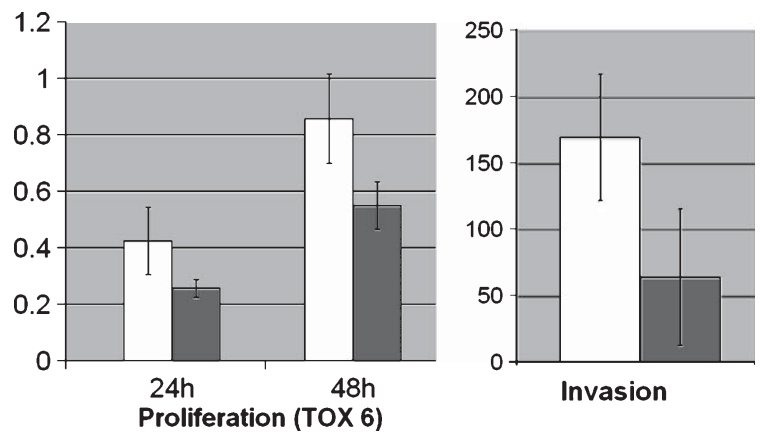

Fig. 2. Left) Changes in the proliferation rates (TOX-6) of recultured cells from the intraocular tumor of animals treated with Imatinib mesylate $(y$-axis $=$ Optical density). Differences measured after 24 and $48 \mathrm{~h}$ were both significant $(P<0.001)$. Right $)$ The invasive abilities of these cells were also significantly impaired ( $y$-axis $=$ percentage invasion $)$. White column, control group. Black column, treated group. ing that the apparent survival advantage in patients diagnosed before the onset of symptoms is seen only in the first year after diagnosis of metastasis. By the second year, cumulative metastatic rates are $90 \%$ or higher in both groups [17]. Several groups attempted different therapeutic approaches to selected groups of patients. Systemic therapies of liver metastases produced a response rate of less than $1 \%$ [18]. Metastectomy of localized nodules $[19,20]$, hepatic artery infusion [21-23], chemoembolization [22, 24, 25] and multi-drug systemic chemotherapy [26-29] have also been tried but no therapy to date could indubitably change the natural history of the disease. Augsburger et al. [1] have recently reviewed in depth the available literature regarding the treatment of metastatic UM and no randomized phase III clinical trials comparing any treatment against observation have been reported. The apparent prolonged survival presented by some centers

Table 1

Changes in gene expression after treatment with Imatinib mesylate in an animal model of uveal melanoma

\begin{tabular}{lccc}
\hline \multicolumn{2}{c}{ Downregulated } & \multicolumn{2}{c}{ Upregulated } \\
Gene & Fold change & Gene & Fold change \\
\hline COL18A1 & -44.51 & SERPINB5 & 290.82 \\
SNCG & -30.33 & CCNE1 & 10.88 \\
NME4 & -11.52 & KISS1 & 10.04 \\
IGF1 & -11.05 & MMP10 & 5.49 \\
PDGFA & -8.36 & HPSE & 5.49 \\
FOS & -6.90 & CDC25A & 5.28 \\
EPDR1 & -6.80 & RORB & 5.27 \\
MCAM & -5.54 & ITGA2 & 5.07 \\
JUN & -4.62 & MMP7 & 4.92 \\
VEGFA & -4.03 & MMP13 & 4.59 \\
TIMP3 & -3.73 & BRCA1 & 4.30 \\
FGFR2 & -3.71 & SSTR2 & 4.22 \\
MYC & -3.65 & GZMA & 3.79 \\
S100A4 & -3.64 & TNF & 3.23 \\
BCL2L1 & -3.53 & HGF & 3.22 \\
MTSS1 & -3.42 & PNN & 3.19 \\
MYC & -3.34 & MYCL1 & 2.84 \\
BAD & -3.18 & CCL7 & 2.79 \\
CDKN2A & -3.17 & CDH1 & 2.79 \\
ITGB5 & -3.16 & CTSL1 & 2.79 \\
IFNA1 & -3.12 & CXCR4 & 2.79 \\
TNFRSF1A & -3.00 & KISS1 R & 2.79 \\
IFNB1 & -2.91 & MTSS1 & 2.79 \\
IL18 & -2.81 & SYK & 2.79 \\
CDKN1A & -2.69 & TIMP4 & 2.79 \\
TP53 & -2.54 & CDK2 & 2.78 \\
AKT1 & -2.38 & IL8RB & 2.51 \\
ITGA3 & -2.37 & PLAUR & 2.49 \\
PDGFB & -2.28 & MMP3 & 2.36 \\
CD82 & -2.16 & CASP8 & 2.35 \\
\hline
\end{tabular}


is likely the result of selection, surveillance or publication biases. As a result, there is a need to develop new effective therapies for metastatic UM.

IM is a tyrosine kinase inhibitor of the Abelson kinase (ABL) that also inhibits the receptor tyrosine kinases (RTKs) KIT and platelet-derived growth factor receptor [30]. IM has been approved by the Food and Drug Administration (FDA) to treat c-kit positive GIST and Philadelphia-chromosome-positive chronic myelogenous leukemia [31]. Response rates are as high as $90 \%$ for those two malignancies [30]. Treatment with IM is generally well tolerated with a low incidence of severe side effects. The most common adverse events include mild to moderate edema, muscle cramps, diarrhea, nausea, skin rashes, and myelosuppression [32]. $\mathrm{C}$-kit is also considered a potential target in a wide variety of other human malignancies where it is expressed and associated with prognosis [5].

To date, the potential use of IM for metastatic UM has been linked to c-kit as the molecular target. That resulted from studies showing high expression of c-kit in primary and metastatic UM [5,33], even though c-kit mutations have not been found [33, 34]. In vitro studies provided more information implicating c-kit in the tumorigenesis of UM [2] and that the c-kit molecular pathway may be important in UM growth [4]. However, data on human patients is rather limited. The first attempt to treat five cases of metastatic UM showed modest results in c-kit positive patients [35], while negative patients showed disease progression. A more recent trial on 12 patients with advanced UM did not observe any objective response [33].

Nonetheless, our findings not only support the idea that IM is still a promising drug for the treatment of metastatic melanoma, but also provide some insight into why previous trials did not succeed. In our animal model, the treatment with IM caused a 10-fold upregulation of KISS1, which was identified as a human melanoma metastasis suppressor gene [36]. We treated additional UM cell lines in vitro and confirmed the upregulation of KISS1 by IM (data not shown). Supporting the role of KISS1 as a MSG, other investigators showed that transfection of KISS1 into metastatic human melanoma cell lines suppressed metastasis in athymic nude mice by $95 \%$ [37]. The role of KISS1 in preventing metastases seems to be related to keeping cells in their dormant state [38]. Therefore, using IM after the detection of macrometastasis is unlikely to cause any impact on the final outcome. By then, cells have already "awaken" from their dormant state, and
KISS 1 would not play a significant role anymore. Interestingly, KISS1 is indeed related to prognosis in UM: Decreased expression of KISS1 in primary UM is associated with decreased survival and higher metastatic rates [39]. The upregulation of a metastasis suppressor gene by IM was an interesting finding that is currently being studied at our laboratory. The drug might be able to prolong the dormancy phase of the disease and finally make a positive impact in the prognosis.

Moreover, IM also altered the expression of other genes that are implicated in the formation of neovessels, which are essential for the evolution of the pre-metastatic niche. On the group of downregulated genes we found Collagen XVIII, a precursor of endostatin, whose high expression has been correlated with poor outcome in lung cancer [40]. PDGFA, PDGFB and $V E G F A$, all known angiogenic factors that play a critical role in cancer angiogenesis [41, 42], were also downregulated. For the group of genes that were upregulated, there was a striking 290-fold increased expression of SERPINB5, also known as Maspin. Maspin (mammary serine protease inhibitor) is a 42 $\mathrm{kDa}$ protein and belongs to the serine protease inhibitor superfamily [43]. The tumor-suppressive function of Maspin is due to inhibition of motility, invasiveness, angiogenesis, and increased sensitivity to apoptosis [44]. The expression of Maspin is higher in lowrisk gastrointestinal stromal tumors when compared to high-risk patients [45]. For cutaneous melanoma, maspin expression was correlated with decreased tumor vessel density and thickness and the expression is lost during the transition from radial to vertical growth phase [46]. As a result, maspin is considered as a tumor suppressor in melanoma by impairing tumor angiogenesis. Aside from impairing neovascularization, IM also caused the downregulation of genes associated with resistance to anticancer drugs and evasion of apoptosis, namely, IGF1 and BCL2L1 [47, 48].

We do acknowledge that our findings do not answer the question whether c-kit or other tyrosine kinases are involved or not in the oncogenesis of UM. Additionally, we cannot assure that IM will be successful for the treatment of patients with advanced metastatic disease. Currently, UM is considered a systemic disease in which cells have already escaped the primary site by the time the primary lesion is diagnosed. The interesting finding of our study is that we revealed another mechanism of action for IM, independent of ckit expression or mutation in UM cells. In keeping with our findings, other investigators confirmed that IM tar- 
gets PDGF signaling in cancer cells and reduces tumor growth. Not only tumor cells but also host smooth muscle neighboring cells (SMC) are affected by IM translating into a decrease in tumor microvessel density and number of SMC-presenting vessels [49]. The result is that not only the "seed" but also the "soil" are changed in a way that impairs the development of the metastatic foci. Therefore, despite disappointing initial results for the treatment of human patients with metastatic melanoma $[33,35]$ we still consider IM as a potential therapy and further research is warranted. We would suggest that future trials be done with patients that are at high-risk of developing metastatic disease, but have not yet presented clinical evidence of such. High-risk UM patients can be identified based on chromossome mutations and genetic signatures in addition to the conventional clinical, histopathological and immunohistochemical prognostic factors. Offering IM treatment to terminal patients is unlikely to change the course of the disease.

In summary, the treatment with IM correlated with fewer and smaller primary tumors as well as less metastatic disease. The previously demonstrated in vitro effects of IM were confirmed in this animal model, specifically lower proliferation and invasion rates for the recultured cells. Interestingly, IM altered the expression of genes known to be related to metastasis but previously not attributed to the action of IM. The upregulation of KISS1 and Maspin, and the downregulation of several proangiogenic factors by the systemic administration of IM may prove beneficial during the preclinical stage of metastatic UM by maintaining microscopic metastatic cells dormant and thereby prolonging "disease-free" survival.

\section{References}

[1] J.J. Augsburger, Z.M. Correa and A.H. Shaikh, Effectiveness of treatments for metastatic uveal melanoma, Am J Ophthalmol 148(1) (2009), 119-127.

[2] G. Lefevre, A.L. Glotin, A. Calipel, et al., Roles of stem cell factor/c-Kit and effects of Glivec/STI571 in human uveal melanoma cell tumorigenesis, J Biol Chem 279(30) (2004), 31769-31779.

[3] M.W. Deininger and B.J. Druker, Specific targeted therapy of chronic myelogenous leukemia with imatinib, Pharmacol Rev 55(3) (2003), 401-423.

[4] C. All-Ericsson, L. Girnita, A. Muller-Brunotte, et al., c-Kitdependent growth of uveal melanoma cells: a potential therapeutic target? Invest Ophthalmol Vis Sci 45(7) (2004), 2075-2082.
[5] P.R. Pereira, A.N. Odashiro, J.C. Marshall, et al., The role of c-kit and imatinib mesylate in uveal melanoma, J Carcinog 4 (2005), 19.

[6] P.L. Blanco, J.C. Marshall, E. Antecka, et al., Characterization of ocular and metastatic uveal melanoma in an animal model, Invest Ophthalmol Vis Sci 46(12) (2005), 4376-4382.

[7] J.C. Marshall, B.F. Fernandes, S. Di Cesare, et al., The use of a cyclooxygenase-2 inhibitor (Nepafenac) in an ocular and metastatic animal model of uveal melanoma, Carcinogenesis 28(9) (2007), 2053-2058.

[8] S. Di Cesare, S. Maloney, B.F. Fernandes, et al., The effect of blue light exposure in an ocular melanoma animal model, $J$ Exp Clin Cancer Res 28 (2009), 48.

[9] G. Blanco, A.M. Saornil, E. Domingo, et al., Uveal melanoma model with metastasis in rabbits: effects of different doses of cyclosporine A, Curr Eye Res 21(3) (2000), 740-747.

[10] I. De Waard-Siebinga, D.J. Blom, M. Griffioen, et al., Establishment and characterization of an uveal-melanoma cell line, Int J Cancer 62(2) (1995), 155-161.

[11] P. Skehan, R. Storeng, D. Scudiero, et al., New colorimetric cytotoxicity assay for anticancer-drug screening, J Natl Cancer Inst 82(13) (1990), 1107-1112.

[12] J.K. Woodward, S.R. Elshaw, A.K. Murray, et al., Stimulation and inhibition of uveal melanoma invasion by HGF, GRO, IL-1 alpha and TGF-beta, Invest Ophthalmol Vis Sci 43(10) (2002), 3144-3152.

[13] M. Diener-West, S.M. Reynolds, D.J. Agugliaro, et al., Development of metastatic disease after enrollment in the COMS trials for treatment of choroidal melanoma: Collaborative Ocular Melanoma Study Group Report No. 26, Arch Ophthalmol 123(12) (2005), 1639-1643.

[14] L. Kodjikian, J.D. Grange, S. Baldo, et al., Prognostic factors of liver metastases from uveal melanoma, Graefes Arch Clin Exp Ophthalmol 243(10) (2005), 985-993.

[15] P. Rietschel, K.S. Panageas, C. Hanlon, et al., Variates of survival in metastatic uveal melanoma, J Clin Oncol 23(31) (2005), 8076-8080.

[16] M. Rivoire, L. Kodjikian, S. Baldo, et al., Treatment of liver metastases from uveal melanoma, Ann Surg Oncol 12(6) (2005), 422-428.

[17] I.K. Kim, A.M. Lane and E.S. Gragoudas, Survival in patients with presymptomatic diagnosis of metastatic uveal melanoma, Arch Ophthalmol 128(7) (2010), 871-875.

[18] A.Y. Bedikian, S.S. Legha, G. Mavligit, et al., Treatment of uveal melanoma metastatic to the liver: a review of the M. D. Anderson Cancer Center experience and prognostic factors, Cancer 76(9) (1995), 1665-1670.

[19] T. Aoyama, M.J. Mastrangelo, D. Berd, et al., Protracted survival after resection of metastatic uveal melanoma, Cancer 89(7) (2000), 1561-1568.

[20] T.M. Pawlik, D. Zorzi, E.K. Abdalla, et al., Hepatic resection for metastatic melanoma: distinct patterns of recurrence and prognosis for ocular versus cutaneous disease, Ann Surg Oncol 13(5) (2006), 712-720.

[21] S.L. Noter, J. Rothbarth, M.E. Pijl, et al., Isolated hepatic perfusion with high-dose melphalan for the treatment of uveal melanoma metastases confined to the liver, Melanoma Res 14(1) (2004), 67-72. 
[22] K. Patel, K. Sullivan, D. Berd, et al., Chemoembolization of the hepatic artery with BCNU for metastatic uveal melanoma: results of a phase II study, Melanoma Res 15(4) (2005), 297-304.

[23] S. Peters, V. Voelter, L. Zografos, et al., Intra-arterial hepatic fotemustine for the treatment of liver metastases from uveal melanoma: experience in 101 patients, Ann Oncol 17(4) (2006), 578-583.

[24] G.M. Mavligit, C. Charnsangavej, C.H. Carrasco, et al., Regression of ocular melanoma metastatic to the liver after hepatic arterial chemoembolization with cisplatin and polyvinyl sponge, JAMA 260(7) (1988), 974-976.

[25] T. Vogl, K. Eichler, S. Zangos, et al., Preliminary experience with transarterial chemoembolization (TACE) in liver metastases of uveal malignant melanoma: local tumor control and survival, J Cancer Res Clin Oncol 133(3) (2007), 177-184.

[26] T. Kivela, S. Suciu, J. Hansson, et al., Bleomycin, vincristine, lomustine and dacarbazine (BOLD) in combination with recombinant interferon alpha-2b for metastatic uveal melanoma, Eur J Cancer 39(8) (2003), 1115-1120.

[27] P.A. O'Neill, M. Butt, C.V. Eswar, et al., A prospective single arm phase II study of dacarbazine and treosulfan as first-line therapy in metastatic uveal melanoma, Melanoma Res 16(3) (2006), 245-248.

[28] S. Pyrhonen, M. Hahka-Kemppinen, T. Muhonen, et al., Chemoimmunotherapy with bleomycin, vincristine, lomustine, dacarbazine (BOLD), and human leukocyte interferon for metastatic uveal melanoma, Cancer 95(11) (2002), 2366-2372.

[29] A. Schmittel, R. Schuster, N.E. Bechrakis, et al., A two-cohort phase II clinical trial of gemcitabine plus treosulfan in patients with metastatic uveal melanoma, Melanoma Res 15(5) (2005), 447-451.

[30] F. Stegmeier, M. Warmuth, W.R. Sellers, et al., Targeted cancer therapies in the twenty-first century: lessons from imatinib, Clin Pharmacol Ther 87(5) (2010), 543-552.

[31] M.H. Cohen, J.R. Johnson and R. Pazdur, U.S. Food and Drug Administration Drug Approval Summary: conversion of imatinib mesylate (STI571; Gleevec) tablets from accelerated approval to full approval, Clin Cancer Res 11(1) (2005), 12-19.

[32] C.F. Waller, Imatinib mesylate, Recent Results Cancer Res 184 (2010), 3-20.

[33] U.B. Hofmann, C.S. Kauczok-Vetter, R. Houben, et al., Overexpression of the KIT/SCF in uveal melanoma does not translate into clinical efficacy of imatinib mesylate, Clin Cancer Res 15(1) (2009), 324-329.

[34] M. Pache, K. Glatz, D. Bosch, et al., Sequence analysis and high-throughput immunohistochemical profiling of KIT (CD 117) expression in uveal melanoma using tissue microarrays, Virchows Arch 443(6) (2003), 741-744.
[35] G. Fiorentini, S. Rossi, G. Lanzanova, et al., Tyrosine kinase inhibitor imatinib mesylate as anticancer agent for advanced ocular melanoma expressing immunoistochemical C-KIT (CD 117): preliminary results of a compassionate use clinical trial, J Exp Clin Cancer Res 22(4 Suppl) (2003), 17-20.

[36] J.H. Lee, M.E. Miele, D.J. Hicks, et al., KiSS-1, a novel human malignant melanoma metastasis-suppressor gene, J Natl Cancer Inst 88(23) (1996), 1731-1737.

[37] J.H. Lee and D.R. Welch, Identification of highly expressed genes in metastasis-suppressed chromosome 6/human malignant melanoma hybrid cells using subtractive hybridization and differential display, Int J Cancer 71(6) (1997), 1035-1044.

[38] C.E. Horak, J.H. Lee, J.C. Marshall, et al., The role of metastasis suppressor genes in metastatic dormancy, APMIS 116(7-8) (2008), 586-601

[39] C.M. Martins, B.F. Fernandes, E. Antecka, et al., Expression of the metastasis suppressor gene KISS 1 in uveal melanoma, Eye 22(5) (2008), 707-711.

[40] H. Chang, T. Iizasa, K. Shibuya, et al., Increased expression of collagen XVIII and its prognostic value in nonsmall cell lung carcinoma, Cancer 100(8) (2004), 1665-1672.

[41] H. Kawagishi, H. Nakamura, M. Maruyama, et al., ARF suppresses tumor angiogenesis through translational control of VEGFA mRNA, Cancer Res 70(11) (2010), 4749-4758.

[42] D. George, Targeting PDGF receptors in cancer - rationales and proof of concept clinical trials, Adv Exp Med Biol 532 (2003), 141-151.

[43] Z. Zou, A. Anisowicz, M.J. Hendrix, et al., Maspin, a serpin with tumor-suppressing activity in human mammary epithelial cells, Science 263(5146) (1994), 526-529.

[44] S. Sheng, A role of novel serpin maspin in tumor progression: the divergence revealed through efforts to converge, $J$ Cell Physiol 209(3) (2006), 631-635.

[45] S.B. Adim, G. Filiz, O. Kanat, et al., Maspin expression in gastrointestinal stromal tumors, World J Surg Oncol 8 (2010), 22.

[46] R. Chua, S. Setzer, B. Govindarajan, et al., Maspin expression, angiogenesis, prognostic parameters, and outcome in malignant melanoma, J Am Acad Dermatol 60(5) (2009), 758-766.

[47] C. Hilmi, L. Larribere, S. Giuliano, et al., IGF1 promotes resistance to apoptosis in melanoma cells through an increased expression of BCL2, BCL-X(L), and survivin, J Invest Dermatol 128(6) (2008), 1499-1505.

[48] J.T. Lee, P. Brafford and M. Herlyn, Unraveling the mysteries of IGF-1 signaling in melanoma, $J$ Invest Dermatol 128(6) (2008), 1358-1360.

[49] A. Pirraco, P. Coelho, A. Rocha, et al., Imatinib targets PDGF signaling in melanoma and host smooth muscle neighboring cells, J Cell Biochem 111 (2) (2010), 433-441. 


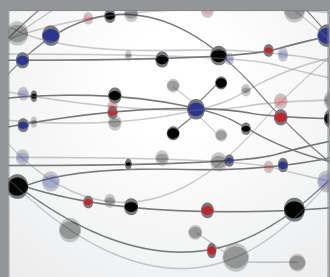

The Scientific World Journal
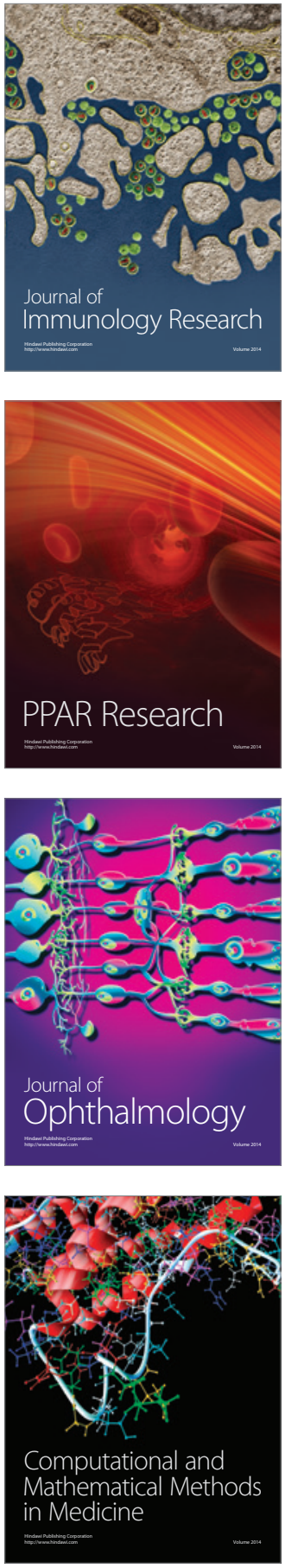

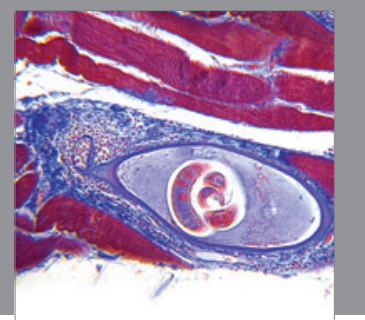

Gastroenterology

Research and Practice
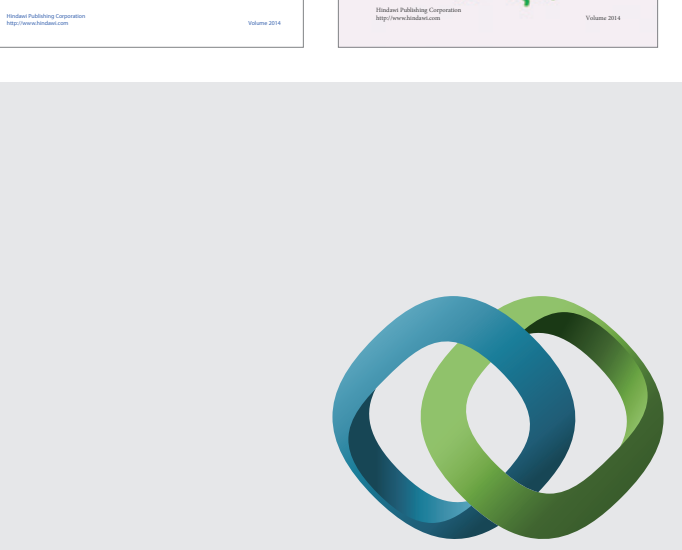

\section{Hindawi}

Submit your manuscripts at

http://www.hindawi.com
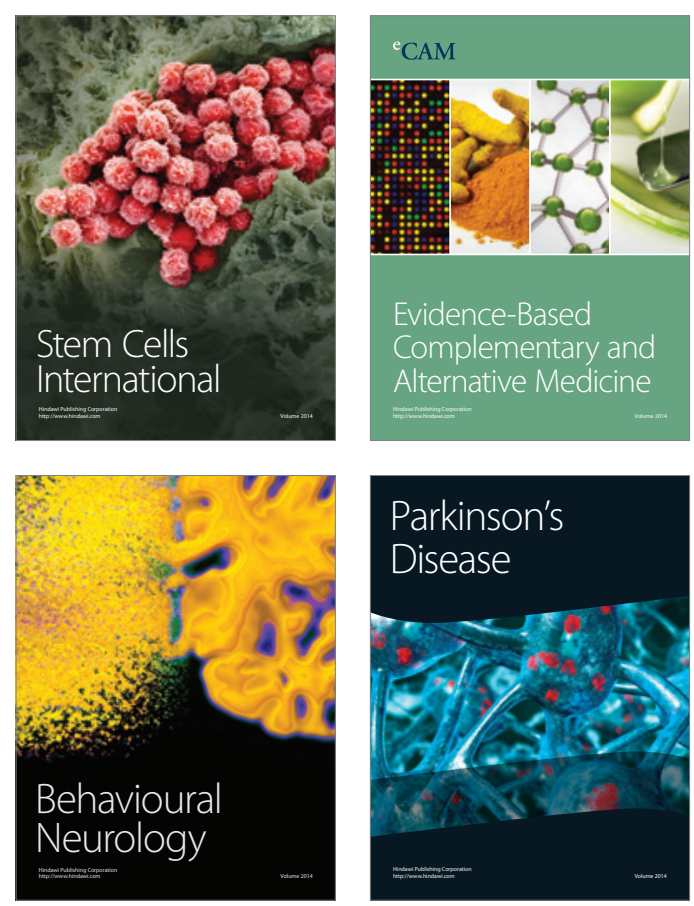

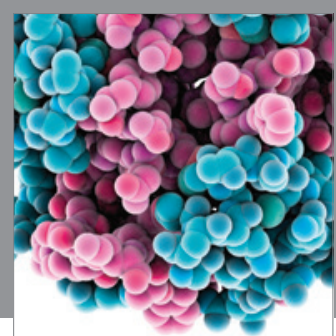

Journal of
Diabetes Research

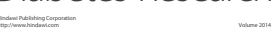

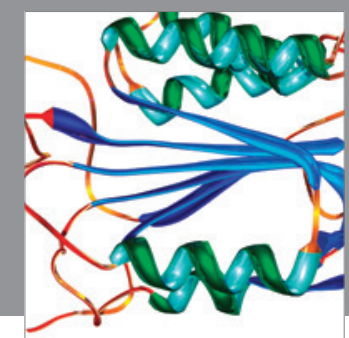

Disease Markers
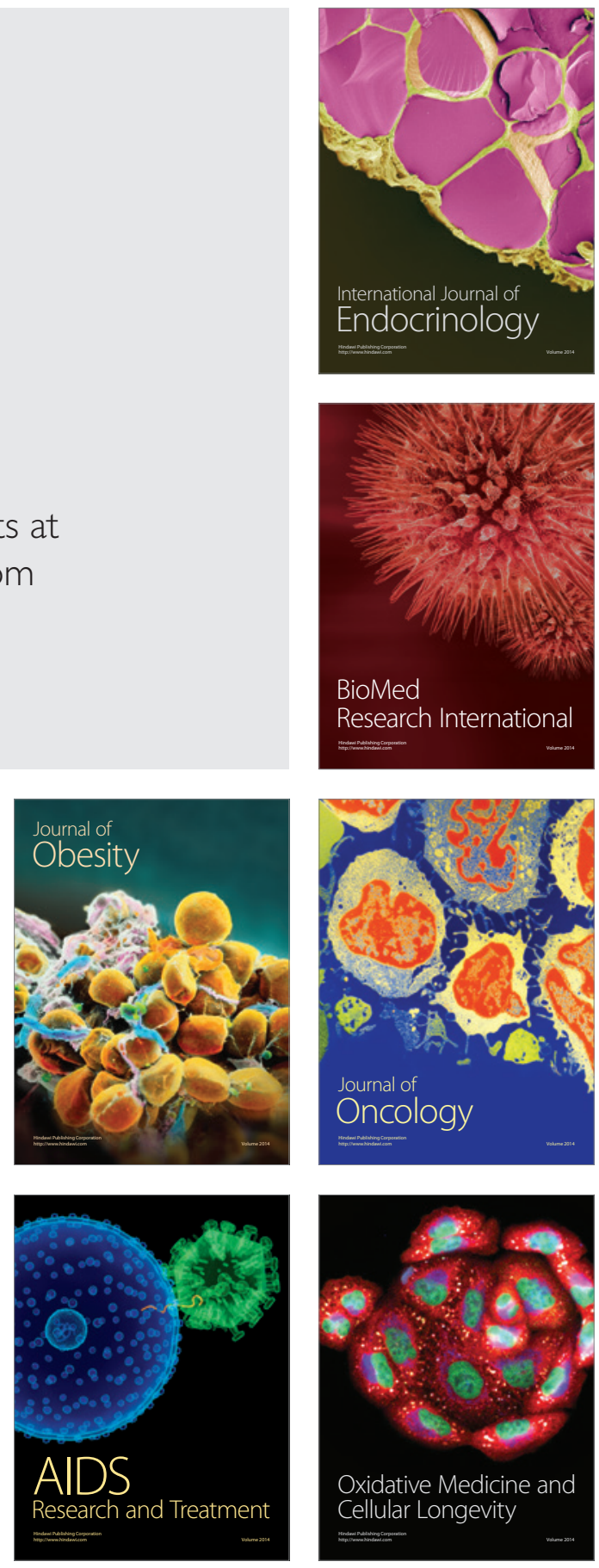